\title{
Possible evolution of dim radio quiet neutron star 1E 1207.4-5209 based on a B-decay model
}

\author{
Aşkın Ankay ${ }^{*}$ Arzu Mert Ankay ${ }^{\dagger}$ Enise Nihal Ercan ${ }^{\ddagger}$ \\ Boğaziçi University \\ Department of Physics \\ 34342-Bebek, İstanbul, Turkey
}

August 19, 2021

\begin{abstract}
Dim radio-quiet neutron star (DRQNS) 1E 1207.4-5209 is one of the most heavily examined isolated neutron stars. Wide absorption lines were observed in its spectrum obtained by both XMM-Newton and Chandra X-ray satellites. These absorption lines can be interpreted as a principal frequency centered at $0.7 \mathrm{keV}$ and its harmonics at $1.4,2.1$ and possibly $2.8 \mathrm{keV}$. The principal line can be formed by resonant proton cyclotron scattering leading to a magnetic field which is two orders of magnitude larger than the perpendicular component of the surface dipole magnetic field (B) found from the rotation period $(\mathrm{P})$ and the time rate of change in the rotation period $(\dot{\mathrm{P}})$ of $1 \mathrm{E} 1207.4-$ 5209. Besides, age of the supernova remnant (SNR) G296.5+10.0 which is physically connected to $1 \mathrm{E} 1207.4-5209$ is two orders of magnitude smaller than the characteristic age $(\tau=\mathrm{P} / 2 \dot{\mathrm{P}})$ of the neutron star. These huge differences between the magnetic field values and the ages can be explained based on a B-decay model. If the decay is assumed to be exponential, the characteristic decay time turns out to be several thousand years which is three orders of magnitude smaller
\end{abstract}

*askin.ankay@boun.edu.tr

†arzu.mert@boun.edu.tr

†ercan@boun.edu.tr 
than the characteristic decay time of radio pulsars represented in an earlier work. The lack of detection of radio emission from DRQNSs and the lack of point sources and pulsar wind nebulae in most of the observed SNRs can also be partly explained by such a very rapid exponential decay. The large difference between the characteristic decay times of DRQNSs and radio pulsars must be related to the differences in the magnetic fields, equation of states and masses of these isolated neutron stars.

Keywords: Neutron star; pulsar: 1E 1207.4-5209; evolution.

\section{Introduction}

Today, several different types of isolated neutron stars which have comparably very different physical properties are known. These are radio pulsars, dim radio-quiet neutron stars (DRQNSs), dim thermal neutron stars (DTNSs), anomalous X-ray pulsars (AXPs) and soft gamma-ray repeaters (SGRs). About 1700 radio pulsars have been observed up to date ${ }^{1}$, some of which have also been detected in other bands of the electromagnetic spectrum. Radio pulsar is relatively the most well known and understood object both theoretically and observationally among all the classes of isolated neutron stars.

DRQNSs, DTNSs and AXPs-SGRs are new types of isolated neutron stars (point X-ray sources). All AXPs-SGRs, which should be considered as a single class, and some DRQNSs and DTNSs, which are cooling neutron stars with short ( $\mathrm{P} \sim$ few 100 milliseconds) and long ( $\mathrm{P} \sim$ few seconds) rotation periods respectively, show themselves as X-ray pulsars. AXPs and SGRs (which have also long rotation periods similar to DTNSs and which experience X-ray and $\gamma$-ray bursts) may be in early phases of at least some of the DTNSs based on field decay models (see Fig.1). Recently, Ankay et al. ${ }^{2}$ have shown the possibility that radio-quiet strong X-ray pulsar J18460258 may be in a phase preceding AXP-SGR and/or DTNS phases based on a detailed comparison between the observational characteristics of these sources.

None of the DRQNSs, DTNSs or AXPs/SGRs has been detected at radio frequencies with the exception AXP XTE J1810-197 from the direction of which radio emission at $1.4 \mathrm{GHz}$ has been detected ${ }^{3}$, but this radio emission may also be produced by a possible pulsar wind nebula (PWN) around this AXP.

There are only a few observationally known DRQNSs. All of them are physically connected to Galactic supernova remnants (SNRs) which have ages 
less than about $20 \mathrm{kyr}^{4,5}$.

There are two basic observable quantities of isolated pulsars: the rotation period $\mathrm{P}$ and the time rate of change of the rotation period $\dot{\mathrm{P}}$. Time rate of change of the rotational kinetic energy of a rigid body is

$$
\dot{E}=I \Omega \dot{\Omega}=\frac{4 \pi^{2} I \dot{P}}{P^{3}}
$$

where $\mathrm{I}$ is the moment of inertia and $\Omega=2 \pi / \mathrm{P}$ is the rotational velocity. The component of the dipole magnetic field of pulsar which is perpendicular to the rotation axis also depends on $\mathrm{P}$ and $\dot{\mathrm{P}}$ :

$$
B=\left(\frac{3 c^{3} I P \dot{P}}{8 \pi^{2} R^{6}}\right)^{1 / 2}
$$

where $\mathrm{c}$ is the speed of light in free space and $\mathrm{R}$ is the radius of pulsar.

If we assume that the time rate of change of the rotational velocity of pulsar can be expressed as a power law:

$$
\dot{\Omega}=-k \Omega^{n}
$$

where $\mathrm{k}$ is a proportionality constant and the power $\mathrm{n}$ is called the 'braking index', then the real age of pulsar can be represented in terms of $\mathrm{P}$ and $\dot{\mathrm{P}}$ as:

$$
t=\frac{P}{(n-1) \dot{P}}\left[1-\left(\frac{P_{0}}{P}\right)^{n-1}\right]
$$

where $\mathrm{P}_{0}$ is the initial rotation period of pulsar. If $\mathrm{P}_{0}$ is much less than $\mathrm{P}$ :

$$
t \cong \frac{P}{(n-1) \dot{P}}
$$

Magneto-dipole radiative power of pulsars is

$$
L=\frac{B_{p}^{2} R^{6} \Omega^{4}}{6 c^{3}} \operatorname{Sin}^{2} \alpha
$$

where $\mathrm{B}_{p}$ is the strength of the dipole magnetic field at the magnetic pole and $\alpha$ is the angle between the rotation axis and the magnetic axis ${ }^{6}$. If $\dot{\mathrm{E}}$ (eqn.(1)) is equal to L (eqn.(6)) (i.e. if the net torque on pulsar is equal to the magneto-dipole radiation torque), then the braking index turns out to be $n=3$ (assuming also that eqn.(3) can be applied to express the rotational evolution of pulsar). In such a case, the characteristic age $(\tau)$ of pulsar is defined using the $\mathrm{n}=3$ condition in eqn.(5):

$$
\tau \cong \frac{P}{2 \dot{P}}
$$


So, when $\mathrm{n}=3$ and $\mathrm{P}_{0} \ll \mathrm{P}, \tau$ is approximately equal to the real age of pulsar. When $\mathrm{n}$ is greater (less) than $3, \tau$ is greater (less) than the real age. So, if there are extra torques on pulsar which spin it down in addition to the effect of the magneto-dipole radiation torque, the pulsar will evolve with larger $\dot{\mathrm{P}}$ values compared to the case of pure magneto-dipole radiation torque. On the other hand, if there is B-decay, the $\dot{\mathrm{P}}$ values the pulsar has throughout the evolution will be smaller. Note that the braking index $\mathrm{n}$ should be adapted as the average braking index $(\bar{n})$ of pulsar when considering long time intervals in pulsar's lifetime, because the value of 'instantaneous' (i.e. considering very short time intervals compared to the lifetime of pulsar) braking index may change in time in a complicated way in general.

Although, none of the DRQNSs has been detected at radio frequencies, this does not necessarily mean that they have no radio emission. There are basically two selection effects (other than the background radiation which is effective only on the sources located in the Galactic central directions, see Ankay et al. ${ }^{7}$ ) which can prevent detection of the radio emission: the beaming fraction and the luminosity function. Based on radio pulsar observations, there exists a strong evidence that beaming fraction decreases in time possibly because of the angle between the rotation axis and the magnetic axis $(\alpha)$ decreasing during the evolution and the large number of the observed radio pulsars with possibly small values of $\alpha$ supports this interpretation ${ }^{8}$. Indeed, the existence of B-decay (where B is the component of the dipole magnetic field perpendicular to the rotation axis) for a large sample of radio pulsars was shown by Guseinov et al. ${ }^{9}$ by comparing the characteristic ages of radio pulsars with their kinematic ages (i.e. their distances from the Galactic plane). Guseinov et al. ${ }^{9}$ assumed an exponential decay with a characteristic decay time $\tau_{d}=3 \times 10^{6} \mathrm{yr}$. The cause of B-decay in the case of radio pulsars can be a temporal decrease in $\alpha$, but the possibility of a decay in the dipole magnetic field itself can not be totally excluded (see e.g Geppert $\&$ Rheinhardt ${ }^{10}$ on the possibility of magnetic field decay in neutron stars).

The other selection effect is related to radio luminosity versus number distribution of radio pulsars (luminosity function). Based on the observational data, most of the radio pulsars must have low radio luminosity at birth and the radio luminosity, which is only a small fraction of the magneto-dipole radiation produced by the pulsar, does not change significantly in time ${ }^{11}$.

As mentioned above, all the known DRQNSs are connected to Galactic SNRs with ages $\leq 2 \times 10^{4} \mathrm{yr}$. Most of the Galactic SNRs are shell-type ${ }^{12-15}$ and many of these SNRs lack detected point sources or PWNe in them. Direct detection of neutron stars in many of the SNRs may not be possible because of the selection effects and the large distances. On the other hand, PWN is not seen around pulsars which have rate of rotational energy loss 
$\dot{\mathrm{E}}<10^{35} \mathrm{erg} / \mathrm{s}^{5,16,17}$. The only known DRQNS with measured $\mathrm{P}$ and $\dot{\mathrm{P}}$ values is $1 \mathrm{E} 1207.4-5209$ and it has $\dot{\mathrm{E}}=2 \times 10^{34} \mathrm{erg} / \mathrm{s}$. This explains why there is no PWN around 1E 1207.4-5209.

For 1E 1207.4-5209, there are 2 problems based on its position on the P-P diagram (Fig.1): first of all, its present B value is much less than the magnetic field measured from its $0.7 \mathrm{keV}$ proton cyclotron line detected by both Chandra and XMM-Newton. Secondly, the $\tau$ value of this DRQNS is much larger than the age of the SNR which it is physically connected to. Below, we will present a B-decay model in order to clarify these problems and to explain the possible evolution of 1E 1207.4-5209.

In Section 2, the observational data on 1E 1207.4-5209 are represented. In Section 3, an exponential B-decay model is introduced as a possible evolutionary scenario for this DRQNS. In the last section, discussions and conclusions are represented.

\section{The observational data on $1 \mathrm{E}$ 1207.4-5209}

DRQNS 1E 1207.4-5209 is the only known isolated neutron star which have X-ray absorption lines clearly observed in its spectrum. The possible proton cyclotron line (0.7 keV) of $1 \mathrm{E}$ 1207.4-5209 and its harmonics at 1.4 and 2.1 $\mathrm{keV}$ (and possibly at $2.8 \mathrm{keV}$ ) detected by XMM-Newton (260 ks observation ${ }^{18,19}$ ) corresponds to a magnetic field $\sim 1.6 \times 10^{14} \mathrm{G}^{18,19}$. Sanwal et al. ${ }^{20}$ also pointed out the possibility that the 0.7 and $1.4 \mathrm{keV}$ lines (Chandra observations $\sim 60 \mathrm{ks}$ ) could be formed by transitions of singly ionized Helium in a magnetic field $\sim(1.4-1.7) \times 10^{14} \mathrm{G}$. We will adapt $\mathrm{B}_{\text {cyclotron }}=1.6 \times 10^{14} \mathrm{G}$ as the present surface dipole magnetic field of $1 \mathrm{E}$ 1207.4-5209. Note that, if the cyclotron line is interpreted in terms of electrons, the corresponding magnetic field value $\left(\sim 8 \times 10^{10} \mathrm{G}^{18,19}\right)$ is much smaller than its present $\mathrm{B}$ value.

Assuming conventional values of mass and radius for 1E 1207.4-5209 and using its measured $\mathrm{P}=424 \mathrm{~ms}$ and $\dot{\mathrm{P}} \cong 1.4 \times 10^{-14} \mathrm{ss}^{-1}$ values, one finds $\mathrm{B} \cong 2.5 \times 10^{12} \mathrm{G}$. This leads to a very small angle between the magnetic axis and the rotation axis: $\alpha \cong 0^{\circ} .9$. On the other hand, SNR G296.5+10.0, which is physically connected to $1 \mathrm{E} 1207.4-5209$, has age $\mathrm{t}_{S N R} \cong 10 \mathrm{kyr}{ }^{4,5,15}$ and the characteristic age of $1 \mathrm{E} 1207-5209 \tau \cong 480 \mathrm{kyr}$. The difference between $\mathrm{t}_{S N R}$ and $\tau$ leads to a very large average braking index unless the initial rotation period $\left(\mathrm{P}_{0}\right)$ of the pulsar is comparable to its present $\mathrm{P}$ value. If $1 \mathrm{E}$ 1207.4-5209 has evolved with $\mathrm{n}=3$ since it was born, it should have $\mathrm{P}_{0} \cong 420$ ms (see eqn.4). A neutron star born with such a large rotation period is a significant problem for theories on core-collapse supernovae and formation 
of pulsars (see Ardeljan et al. ${ }^{21}$, Moiseenko et al. ${ }^{22}$ and Heger et al. ${ }^{23,24}$ on the necessity of rapid rotation for newborn neutron stars), though this possibility may not be totally excluded.

\section{$3 \quad$ B-decay model for 1E 1207.4-5209}

We can explain the huge difference between $\mathrm{t}_{S N R}$ and $\tau$ and the large discrepancy between $\mathrm{B}_{\text {cyclotron }}$ and $\mathrm{B}$ by a simple B-decay model. We will assume an exponential decay similar to Guseinov et al. ${ }^{9}$ who showed the existence of B-decay for a large sample of radio pulsars by comparing the kinematic ages with the characteristic ages. Assuming conventional values of mass and radius for the pulsar and using eqn.(2), the exponential B-decay can be written as:

$$
P \dot{P}=\frac{B_{0}^{2}}{10^{39}} e^{-2 t / \tau_{d}}
$$

for $\mathrm{t} \gg \tau_{d}$. Here, $\mathrm{P}$ and $\dot{\mathrm{P}}$ are the present values of the pulsar, $\mathrm{B}_{0}$ is the initial value of the magnetic field component perpendicular to the rotation axis, $\tau_{d}$ is the characteristic decay time, and $t$ is the real age of pulsar. If the initial angle $\alpha_{0}=90^{\circ}$ (i.e. if $\mathrm{B}_{0}=\mathrm{B}_{\text {cyclotron }}$ assuming no field decay), then $\tau_{d} \cong 2.4 \times 10^{3}$ yr for $\mathrm{t}=\mathrm{t}_{S N R} \cong 10^{4} \mathrm{yr}$.

We can also put an upper limit on $\tau_{d}$ : if the pulsar had evolved with $\mathrm{n}=3$, it should have had $\mathrm{B}_{0} \cong 1.7 \times 10^{13} \mathrm{G}$ (assuming that the pulsar's age is $10^{4}$ yr and $\mathrm{P}_{0} \ll \mathrm{P}$, see Fig. 1 ). Using $\mathrm{B}_{0}=2 \times 10^{13} \mathrm{G}$ in eqn.(8) gives $\tau_{d} \cong 4.8 \times 10^{3}$ yr. So, $\tau_{d} \cong(2.4-4.8) \times 10^{3}$ yr corresponding to $\mathrm{B}_{0} \cong(16-2) \times 10^{13} \mathrm{G}$. In this analysis, we assume that a possible evolution with $\bar{n}<3$ when the pulsar is very young compared to its present age will have a negligible influence on its evolution on the $\mathrm{P}-\dot{\mathrm{P}}$ diagram.

In the B-decay model introduced above, the characteristic age can be written as a function of $\tau_{d}$ and $\mathrm{t}$ as:

$$
\tau=\frac{\tau_{d}}{2} e^{2 t / \tau_{d}}
$$

with the condition $\mathrm{t} \gg \tau_{d}$. Using eqns. (7),(8) and (9), we have found the positions on the P-P diagram (shown by 'light squares' in Fig.1) corresponding to different evolutionary tracks based on the B-decay model. In Fig.1, the points ('light squares') a $(\mathrm{t}=5 \mathrm{kyr}), \mathrm{b}(\mathrm{t}=10 \mathrm{kyr})$ and $\mathrm{c}(\mathrm{t}=15 \mathrm{kyr})$ are on the evolutionary track with $\mathrm{B}_{0}=2 \times 10^{13} \mathrm{G}$ and $\tau_{d}=2.5 \mathrm{kyr}$. The points $\mathrm{d}$ $(\mathrm{t}=10 \mathrm{kyr})$, e $(\mathrm{t}=15 \mathrm{kyr})$ and $\mathrm{f}(\mathrm{t}=20 \mathrm{kyr})$ correspond to the evolutionary track with $\mathrm{B}_{0}=2 \times 10^{13} \mathrm{G}$ and $\tau_{d}=5 \mathrm{kyr}$. The points $\mathrm{g}(\mathrm{t}=5 \mathrm{kyr}), \mathrm{h}(\mathrm{t}=10$ $\mathrm{kyr})$, $\mathrm{i}(\mathrm{t}=15 \mathrm{kyr})$ and $\mathrm{j}(\mathrm{t}=10 \mathrm{kyr}), \mathrm{k}(\mathrm{t}=15 \mathrm{kyr}), \mathrm{l}(\mathrm{t}=20 \mathrm{kyr})$ are on the 
evolutionary tracks with $\mathrm{B}_{0}=4 \times 10^{13} \mathrm{G}, \tau_{d}=2.5 \mathrm{kyr}$ and $\mathrm{B}_{0}=4 \times 10^{13} \mathrm{G}, \tau_{d}=5$ kyr, respectively.

The present position of $1 \mathrm{E} 1207.4-5209$ on the $\mathrm{P}-\dot{\mathrm{P}}$ diagram is in between the last two tracks $\left(\mathrm{B}_{0}=4 \times 10^{13} \mathrm{G}, \tau_{d}=2.5 \mathrm{kyr}\right.$ and $\mathrm{B}_{0}=4 \times 10^{13} \mathrm{G}, \tau_{d}=5 \mathrm{kyr}$, see Fig.1). We can conclude that $\mathrm{B}_{0} \cong 4 \times 10^{13} \mathrm{G}$ and $\tau_{d} \cong 3.75 \mathrm{kyr}$ for this DRQNS in the B-decay model. The corresponding initial value of the angle between the rotation axis and the magnetic field axis is $\alpha_{0} \cong 14^{\circ} .5$.

\section{Discussions and Conclusions}

If the present surface dipole magnetic field of $1 \mathrm{E} 1207.4-5209$ is about $1.6 \times 10^{14}$ $\mathrm{G}$ based on resonant proton cyclotron scattering measurements and since $\mathrm{B}_{1207}=2.5 \times 10^{12} \mathrm{G}$, there may be a decay in the angle $\alpha$ between the rotation and the magnetic axes (but not a decay in the magnetic field itself) considering the huge difference between the characteristic age of 1E 1207.4-5209 and the age of its SNR. This can be explained by a simple exponential Bdecay model. Characteristic time of the exponential decay must be about 2.5-5 kyr depending on the initial value of $\mathrm{B}$ which must be in the interval $\mathrm{B}_{0}=(16-2) \times 10^{13} \mathrm{G}$. Such a rapid decrease in $\alpha$ leads to a short lifetime (possibly about $(1-2) \times 10^{4}$ yr taking into consideration the ages of the SNRs physically connected to DRQNSs) for this type of neutron star as an X-ray pulsar and this may also be the reason for the lack of detected radio emission from such neutron stars as the radio beam width can be narrower compared to the X-ray beam width. After about $(1-2) \times 10^{4}$ yr pass since the birth of such a pulsar, it can be detected as a cooling neutron star (but not as an X-ray or radio pulsar) if it is located close enough to the Sun. This result can be used to explain the lack of detected point sources in at least some of the Galactic SNRs (especially the distant ones) as the lifetime of an SNR can be as long as $10^{5} \mathrm{yr}$.

The huge difference between the characteristic age of 1207.4-5209 and the age of its SNR may in principle be explained by assuming that this DRQNS was born with an initial rotation period very close to its present rotation period. But such a long initial rotation period $\left(\mathrm{P}_{0} \cong 420 \mathrm{~ms}\right)$ contradicts the estimations based on existing theories on formation of neutron stars by core-collapse supernovae (which predict one order of magnitude smaller $\mathrm{P}_{0}$ values).

On the other hand, the huge difference between the surface dipole magnetic field (about $1.6 \times 10^{14} \mathrm{G}$ obtained from the cyclotron line measurement) of $1 \mathrm{E}$ 1207.4-5209 and its B value (about $2.5 \times 10^{12} \mathrm{G}$ ) can not be explained by assuming a constant and very small initial angle $\left(\alpha_{0} \cong 0^{\circ} .9\right)$ between the 
rotation and magnetic axes, if the initial rotation period is much less than the present rotation period (i.e. if the average braking index is much greater than 3 , see eqn.4 and eqn.5).

There may be basically two different magnetic fields in neutron stars: the magnetic field formed by superconducting entrainment currents which is parallel to the rotation axis and the 'relic' magnetic field (which may be homogeneous inside and in the form of a dipole field on the surface of the neutron star) which is formed when the progenitor star collapses leading to supernova explosion ${ }^{25,26}$. The relic magnetic field axis can have an arbitrary angle $(\alpha)$ with respect to the rotation axis and the resultant magnetic field will be a superposition of the two magnetic fields.

As the axis of the relic magnetic field approaches the rotation axis, the magnitude of the resultant surface magnetic field (i.e. the total magnetic field on the surface which is the superposition of the relic field and the field formed by the superconducting entrainment currents) increases and its component which is perpendicular to the rotation axis decreases in time. Since 1E 1207.4-5209 has most probably very high $\mathrm{B}_{\text {cyclotron }}$ and comparably very low B (perpendicular component of the resultant surface magnetic field) and as this neutron star is observed as a pulsar, $\mathrm{B}_{\text {cyclotron }}$ must be comparable to the resultant surface magnetic field which is increasing in time (or to the relic magnetic field as the magnetic field formed by the entrainment currents must be on the order of $10^{12} \mathrm{G}$ on average ${ }^{25,26}$ ). On the other hand, the actual low $B$ value of 1 E 1207.4-5209 as compared to the case of $n=3$ (see Fig.1) can be explained by the exponential decay as this perpendicular component decreases in time based on the discrepancy between the age values.

The increase in the magnitude of the resultant surface magnetic field of 1E 1207.4-5209 must be very small if the relic field is actually much higher than the entrainment field, so that the $\mathrm{B}_{\text {cyclotron }}$ and hence the magnitude of the present resultant surface magnetic field must be comparable to the magnitude of the initial resultant surface magnetic field. On the other hand, the decrease in the perpendicular component of the resultant surface magnetic field must be very large (about one order of magnitude) in a very short time interval $\left(\sim 10^{4} \mathrm{yr}\right)$ compared to radio pulsar lifetimes. In the case of radio pulsars, the increase in the magnitude of the resultant surface magnetic field must be relatively large (up to a factor of about 1.5) based on the known $B$ values of radio pulsars and the prediction on the average strength of the entrainment field, and the decrease in the perpendicular component of the field must exist on a much longer timescale. Comparing the magnetic field values of $1 \mathrm{E}$ 1207.4-5209 with the $\mathrm{B}$ values of most of the radio pulsars together with the age discrepancy for 1E 1207.4-5209 - SNR G296.5+10.0 pair (which also exists for several other pulsar - SNR pair but without any clearly 
detected cyclotron line) may be an evidence for the existence of two different magnetic fields for neutron stars; the relic field and the entrainment field. The interaction between the superconducting entrainment currents and the relic field may be the reason for the decrease in $\alpha$. Such a possible interaction between the two fields throughout the evolution of neutron stars with different initial intrinsic physical conditions has yet to be examined.

The rate of temporal decrease in $\alpha$ (and hence the evolution of the perpendicular component of the total surface dipole magnetic field) may depend on both the relic and hence the resultant magnetic field, the mass and the equation of state of the neutron star (it may also depend on the initial value of $\alpha$ ). The changes in $\dot{\mathrm{P}}$ observed for 1E 1207.4-5209 may also be explained by oscillations in the magnetic dipole axis as it approaches the rotation axis. Such changes in $\dot{\mathrm{P}}$ must also exist in other 1207-like isolated radio-quiet X-ray pulsars.

As a comparison please note that the magnetic field values of accreting $\mathrm{X}$-ray pulsars in X-ray binaries obtained from cyclotron line measurements are on the order of $10^{12} \mathrm{G}^{27}$, that is comparable to the predicted surface magnetic fields due to the entrainment currents ${ }^{25,26}$, and the B values of recycled millisecond pulsars are several orders of magnitude smaller (see BisnovatyiKogan \& Komberg ${ }^{28,29}$ who gave the first reliable explanation for the magnetic field decay in X-ray binaries by accretion and the formation of recycled millisecond pulsars). The surface magnetic field in the case of X-ray binaries most probably decreases because of the plasma falling upon the surface of the neutron star during the accretion process ${ }^{28-32}$. A decrease in the resultant surface field because of accretion and maybe also a decrease in the value of $\alpha$ (but on a much longer timescale compared to the $1 \mathrm{E}$ 1207.4-5209 case) may explain the measured conventional $10^{12} \mathrm{G}$ magnetic field values of $\mathrm{X}$-ray pulsars in binary systems and the very low perpendicular components of the resultant surface field of recycled millisecond pulsars.

As a last remark we would like to note that the birth rate of 1207-like X-ray pulsars must be about $20-30 \%$ of the total supernova rate (excluding type-Ia supernovae as this type of explosion most probably does not lead to formation of a neutron star) taking into consideration that the lifetime of such sources as X-ray pulsars must be about $2 \times 10^{4}$ yr because of rapid B-decay (see Guseinov et al. ${ }^{5}$ on the birth rates of different types of isolated neutron stars including dim radio quiet neutron stars some of which seem to be 1207-like objects based on the existing observational data).

Acknowledgments We would like to thank the anonymous referee for his/her constructive and helpful contributions. This work is supported by Boğaziçi University. 


\section{References}

1. ATNF - Australia Telescope National Facility Pulsar Catalogue (2006), http://www.atnf.csiro.au/research/pulsar/psrcat/.

2. A. Ankay, S. Sahin, G. Karanfil and E. Yazgan, Int. J. Mod. Phys. D 14, 1075 (2005).

3. J. P. Halpern, E. V. Gotthelf, R. H. Becker, D. J. Helfand and R. L. White, Astrophys. J. 632, L29 (2005).

4. O. H. Guseinov, A. Ankay and S. O. Tagieva, Astrophys. Space Sci. 289, $23(2004)$.

5. O. H. Guseinov, A. Ankay and S. O. Tagieva, Int. J. Mod. Phys D 14, $643(2005)$.

6. V. M. Lipunov, Astrophysics of Neutron Stars, Springer (1992).

7. A. Ankay, O.H. Guseinov and S.O. Tagieva, Astronomical and Astrophysical Transactions 23, 503 (2004).

8. T. M. Tauris and R. N. Manchester, Mon. Not. R. Astron. Soc. 298, 625 (1998).

9. O. H. Guseinov, A. Ankay and S. O. Tagieva, Int. J. Mod. Phys. D 13, 1805 (2004).

10. U. Geppert and M. Rheinhardt, Astronomy and Astrophysics 392, 1015 (2002).

11. O. H. Guseinov, E. Yazgan, S. O. Tagieva and S. Ozkan, Rev. Mex. Astron. Astrof. 39, 267 (2003).

12. D. A. Green, A Catalogue of Galactic Supernova Remnants (2006 April version), http://www.mrao.cam.ac.uk/surveys/snrs/.

13. O. H. Guseinov, A. Ankay and S. O. Tagieva, Serb. Astron. J. 167, 93 (2003).

14. O. H. Guseinov, A. Ankay and S. O. Tagieva, Serb. Astron. J. 168, 55 (2004).

15. O. H. Guseinov, A. Ankay and S. O. Tagieva, Serb. Astron. J. 169, 65 (2004).

16. O. H. Guseinov, A. Ankay, S. O. Tagieva and M. O. Taskin, Int. J. Mod. Phys. D 13, 197) (2004).

17. O. H. Guseinov, A. Ankay and S. O. Tagieva, astro-ph/0401114 (2004).

18. A. De Luca, P. Caraveo, S. Mereghetti, M. Moroni, G. F. Bignami and R. Mignani, Young Neutron Stars and Their Environments, IAU Symposium no.218, edited by Fernando Camilo and Bryan M. Gaensler, p.273 (2004).

19. A. De Luca, S. Mereghetti, P. Caraveo, M. Moroni, R. Mignani and G. F. Bignami, Astron. Astrophys. 418, 625 (2004).

20. D. Sanwal, G. G. Pavlov, V. E. Zavlin and M. A. Teter, Astrophys. J. 574, L61 (2002). 
21. N. V. Ardeljan, G. S. Bisnovatyi-Kogan and S. G. Moiseenko, Mon. Not. R. Astron. Soc. 359, 333 (2005).

22. S. G. Moiseenko, G. S. Bisnovatyi-Kogan, N. V. Ardeljan, Mon. Not. R. Astron. Soc. 370, 501 (2006).

23. A. Heger, S. E. Woosley, N. Langer and H. C. Spruit, Stellar Rotation, proceedings of IAU Symposium No.215, edi. by Andre Maeder and Philippe Eenens, p.591 (2004).

24. A. Heger, S. E. Woosley and H. C. Spruit, Astrophys. J. 626, 350 (2005).

25. D. M. Sedrakian and D. Blaschke, Astrophysics 45, 166 (2002).

26. D. M. Sedrakian and K. M. Shahabasyan, "The Magnetic Field of Pulsars" in Neutron Stars, Supernovae and Supernova Remnants, edi. by Efe Yazgan, Askin Ankay and Oktay H. Guseinov, Nova Science Publishers (to be published in 2006).

27. W. Coburn, W. A. Heindl, R. E. Rothschild, D. E. Gruber, I. Kreykenbohm, J. Wilms, P. Kretschmar and R. Staubert, Astrophys. J. 580, 394 (2002).

28. G. S. Bisnovatyi-Kogan and B. V. Komberg, Sov. Astron. 18, 217 (1974).

29. G. S. Bisnovatyi-Kogan and B. V. Komberg, Sov. Astron. Lett. 2, 130 (1976).

30. G. S. Bisnovatyi-Kogan and A. M. Fridman, Astron. Zh. 46, 721 (1969).

31. P. R. Amnuel and O. H. Guseinov, Astron. Tsirk. No.524 (1969).

32. G. S. Bisnovatyi-Kogan, "Evolutions of Neutron Stars and Their Magnetic Fields" in Neutron Stars, Supernovae and Supernova Remnants, edi. by Efe Yazgan, Askin Ankay and Oktay H. Guseinov, Nova Science Publishers (to be published in 2006). 


\section{Figure Caption}

Rotation period $(\mathrm{P})$ versus temporal change of rotation period $(\dot{\mathrm{P}})$ diagram of various types of isolated pulsars. Small dots represent radio pulsars. Symbols 'cross' and 'star' denote AXPs-SGRs and DTNSs, respectively (upper limits on $\dot{\mathrm{P}}$ for 3 DTNSs are shown by arbitrary arrows). The actual position of $1 \mathrm{E}$ 1207.4-5209 is shown by a 'dark square' and its position for $n=3$ case (see text) is displayed by a 'plus' sign. Constant lines of B, $\dot{\mathrm{E}}$ and $\tau$ are denoted by B11-B15, E29-E41 and T3-T9, respectively. Some points on the evolutionary tracks found from the exponential B-decay model (see text) are displayed as 'light squares': a,b,c $\left(\mathrm{B}_{0}=2 \times 10^{13} \mathrm{G}, \tau_{d}=2.5 \mathrm{kyr}\right)$; d,e,f $\left(\mathrm{B}_{0}=2 \times 10^{13} \mathrm{G}, \tau_{d}=5\right.$ kyr); g,h,i $\left(\mathrm{B}_{0}=4 \times 10^{13} \mathrm{G}, \tau_{d}=2.5 \mathrm{kyr}\right) ; \mathrm{j}, \mathrm{k}, \mathrm{l}\left(\mathrm{B}_{0}=4 \times 10^{13} \mathrm{G}, \tau_{d}=5 \mathrm{kyr}\right)$. 


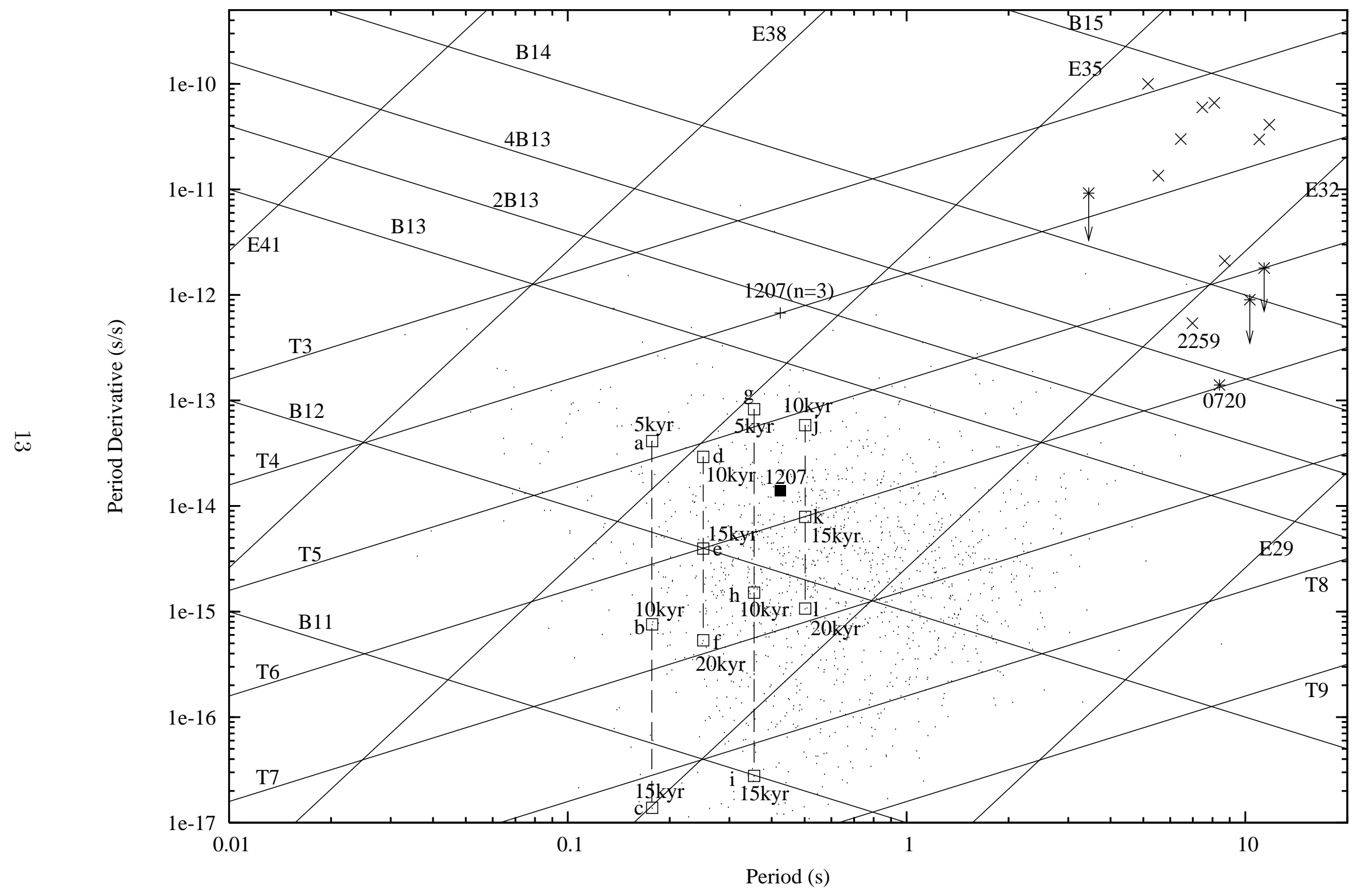

